Original Research Paper

\title{
The Effect of Pulsed Electromagnetic Field Exposure on Fracture Healing Through the Wnt Signal Pathway
}

\author{
${ }^{1,2}$ Umiatin U, ${ }^{3}$ Ismail Hadisoebroto Dilogo, ${ }^{4}$ Puji Sari and ${ }^{5}$ Sastra Kusuma Wijaya \\ ${ }^{I}$ Department of Physics, Faculty of Mathematics and Natural Science, Universitas Negeri Jakarta, Jakarta, Indonesia \\ ${ }^{2}$ Doctoral Program in Biomedical Science, Faculty of Medicine, Universitas Indonesia, Jakarta, Indonesia \\ ${ }^{3}$ Department of Orthopaedic and Traumatology, Cipto Mangunkusumo General Hospital, \\ Faculty of Medicine, Universitas Indonesia, Jakarta, Indonesia \\ ${ }^{4}$ Department of Biology, Faculty of Medicine, Universitas Indonesia, Jakarta, Indonesia \\ ${ }^{5}$ Department of Physics, Faculty of Mathematics and Natural Science, Universitas Indonesia, Depok, Indonesia
}

\begin{abstract}
Article history
Received: 27-08-2020

Revised: 08-12-2020

Accepted: 12-12-2020

Corresponding Author:

Umiatin U.

Department of Physics, Faculty

of Mathematics and Natural

Science, Universitas Negeri

Jakarta, Jakarta, Indonesia

Email: umiatin@unj.ac.id

Ismail Hadisoebroto Dilogo

Department of Orthopaedic and

Traumatology, Cipto

Mangunkusumo General

Hospital, Faculty of Medicine,

Universitas Indonesia, Jakarta,

Indonesia

Email: ismailortho@gmail.com
\end{abstract}

\section{Introduction}

Various strategies have been used to encourage fracture healing, including the use of biological and biochemical methods. Another method is the use of physics-based methods, such as Low-Intensity Pulsed Ultrasound (LIPUS) and Pulsed Electromagnetic Fields (PEMF) that have been widely studied and reported to encourage fracture healing (Kooistra et al., 2009). In a preliminary study using delayed union fracture rat model, PEMF exposure was reported to improve fracture healing based on radiographic examination and RUST score analysis (Dilogo et al., 2018). Other studies suggest that PEMF exposure can induce intracellular calcium uptake in osteoblasts (Zhang et al., 2010).

\begin{abstract}
Pulsed Electromagnetic Fields (PEMF) is reported to encourage the healing of nonunion fractures. However, the mechanism by which this occurs is still not known. Wnt signaling pathways are believed to be important signaling pathways in bone formation. This study will evaluate the healing of delayed union femur fracture, given PEMF exposure. 48 Spraque Dawley rats were facturized at the left femoral shaft. These rats were randomized into two goups: A control group (24 rats) and the PEMF group (24 rats). The PEMF every day for $5,10,18$ and 28 days, while the contre group was not given PEMF exposure. Consequently, on days 5, 10, 18 and 28 days after fracture, 6 rats from each group were sacrificed. Callus bone was used for histological and RT-PCR examination on the expression of Wnt10b, Wnt5a and $\beta$-catenin. Blood samples were taken to examine Alkaline Phosphatase (ALP) activity using the ELISA method. Hematoxylin Eosin (HE) staining results showed that in the initial phase of healing, fibrous tissue in the fracture gap of the PEMF group was less compared to the control group. In the PEMF group, ALP activity increased significantly on day 10 . This is thought to be related to an incre in osteoblast activity in a bone matrix formation. Furthermore, RTexpresamination results showed that Wnt10b, Wnt5a and $\beta$-catenin gene be concluded that PEMF exposure is thought to accelerate delayed union fracture healing through the Wnt signal pathway.
\end{abstract}

Keywords: Fracture, Delayed Union, PEMF, Alkaline Phosphatase, Wnt

PEMF exposure is also reported to be able to maintain the composition and molecular structure of cartilage (Liu et al., 1996). In vitro studies by $\mathrm{Fu}$ et al. reported that single PEMF exposure was able to accelerate osteogenic differentiation from hBMSCs and increase the bone mineral formation and neo-vascularization (Fu et al., 2014). Other pre-clinical studies mention that the use of PEMF in the early wound healing period can increase the rate of healing (Choi et al., 2016; Shi et al., 2013). At the molecular level was found that there was an increase in the expression of bone formation marker genes, including bone sialoprotein, osteopontin, osteonectin and Runx 2 on Bone Marrow Mesenchymal Stem Cell (BM-MSCs) after exposure to PEMF (Ceccarelli et al., 2013). PEMF exposure also led to an increase in microarchitecture 
and bone mechanical strength through the activation of the Wnt/ $\beta$-catenin signaling pathway (Jing et al., 2013). In a study by (Shi et al., 2013), osteogenic differentiation from Adipose-Derived Stem Cells (ADSCs) was found to increase after exposure to PEMF with a frequency of $45 \mathrm{~Hz}$ and inhibited osteoclast formation. PEMF exposure was reported decrease the expression of PPAR $\gamma$ and Adipoq in the adipogenic differentiation of MSCs. (Sari et al., 2020). MSCs is a multipotent cell that can differentiate into several type of cells, including chondrocyte, osteocyte and adipocyte. The other study suggested that there was inverse relationship between osteogenic and adipogenic differentiation of MSCs (Gimble et al., 2006). Various studies both in vitro and in vivo show that PEMF exposure was able to affect the fracture healing process; however, the signaling pathway involved for biochemical signal response and transduction has not been identified until now (Shi et al., 2013).

Fracture healing is a complex process orchestrated by various molecular signaling pathways, including the Wnt signaling pathway. Based on the activity and mechanism of signal transduction, $19 \mathrm{Wnt}$ proteins are classified into two groups, termed canonical and non-canonical. The canonical Wnt signaling pathway, also known as the Wnt/ $\beta$-catenin signaling pathway, has a role in stabilizing $\beta$-catenin in the cytoplasm. Concurrently, the non-canonical Wnt signaling pathways are not associated with $\beta$-catenin stabilization in the cytoplasm. Canonical Wnt proteins include Wnt3A, Wnt8 and Wnt10b, while non-canonical proteins include Wnt5a, Wnt4, Wnt11. (Bao et al., 2017; Chen and Alman, 2009; Marcellini et al., 2012).

The canonical pathway is activated when the Wnt ligand binds to Frizzled (Fzd) receptors; this is followed by the stabilization of $\beta$-catenin in the nucleus. This will then regulate transcription factors in the Wnt target gene. Overexpression of Wnt10b on the canonical pathway has been reported to induce osteoblast differentiation and increase bone mass. In the last decade, the extensively studied canonical Wnt signaling pathway is a potential treatment for the bone disease; however, there is a lack of literature concerning the non-canonical Wnt pathway in bone disease treatment (Heilmann et al., 2013). In this study, we carried out histological, molecular and biochemical studies to determine whether PEMF exposure can promote healing of delayed union fractures via the Wnt signaling pathway.

\section{Materials and Methods}

\section{Experimental Design}

This study used 48 male Spraque Dawley rats (average age 12 weeks; average weight $300 \pm 20 \mathrm{~g}$ ) put randomly into two groups: The control group (24 rats without PEMF exposure) and PEMF group (24 rats with
PEMF exposure). Each group was further divided into four sub-groups, each of which was sacrificed on days 5 , 10, 18 and 28 after fracture (Table 1). All rats were acclimatized for 1 week prior to the preparation of the fracture model. 6 rats were placed in a plastic cage with room temperature $(23 \pm 0.5)^{\circ} \mathrm{C}$, stable air humidity and a natural day/night cycle. The rats had free access to a standard pellet diet and water ad libitum.

\section{Rats Fracture Model}

All rats were anesthetized with ketamine $(70 \mathrm{mg} / \mathrm{kg}$ body weight) and Xylazine (35 mg/kg body weight) administered intraperitoneally as pre-operative treatment. Aseptic and agar antiseptic procedures were done to the left thigh. An incision of approximately 2 $\mathrm{cm}$ in length was performed at the posterolateral side of the left femur in the $1 / 3$ middle of the shaft. The fracture site was exposed and $5 \mathrm{~mm}$ of the periosteum was stripped on each side to create the delayed union model. A $1.2 \mathrm{~mm}$ diameter $\mathrm{K}$-wire was inserted into the femoral canal in a retrograde fashion through the knee joint to stabilize the fracture with the use of a motordriven drill (Fauzi et al., 2015). Lastly, soft tissues were sutured with absorbable sutures at the end of the procedure. Post-operative pain was managed by the administration of the intramuscular dose of penicillin and streptomycin (Kokubu et al., 2003). The study protocol was reviewed and approved by our institutional ethical committee with an approval number 17-05-0489.

\section{PEMF Stimulation}

The PEMF exposure system was generated using paired Helmholtz coil $60 \mathrm{~cm}$ in diameter and placed 30 $\mathrm{cm}$ apart. In PEMF groups, the whole body of rats was exposed to PEMF (1.6 mT, $50 \mathrm{~Hz}$ ) for 4h/day within 128 days. In the control group, animals were not exposed to PEMF. The PEMF device was explained in previous publications (Umiatin et al., 2019).

\section{Histological Evaluation}

Histological analysis was performed on 24 of the 48 samples ( $\mathrm{n}=12$ each for PEMF and control group). The animals were sacrificed at days 5, 10, 18 and 28 post-fracture by a lethal intravenous dose of ketamine and xylazine. Immediately after death, the femurs were excised, soft tissue was dissected. The femurs were then fixed in $10 \%$ neutral buffer formalin for $48 \mathrm{~h}$ by maintaining the $\mathrm{K}$ wire. The specimens were then decalcified with Hydrochloric acid (ShandonTM TBD1TM Rapid Decalcifier, Thermo Scientific). After decalcification, specimens were embedded in paraffin and cut into $5 \mu \mathrm{m}$ thick longitudinal sections. The sections were stained with Hematoxylin and Eosin (HE). Standardized histological images were obtained with Nikon eclipse microscope. 
Table 1: Number of animals in each group

\begin{tabular}{lllll} 
& Control & & PEMF \\
Euthanasia & -- Histology & qRT-PCR & Histology & qRT-PCR \\
\hline 5 days & 3 & 3 & 3 & 3 \\
10 days & 3 & 3 & 3 & 3 \\
18 days & 3 & 3 & 3 & 3 \\
28 days & 3 & 3 & 3 & 3 \\
\hline
\end{tabular}

Table 2: The primer used for RT-qPCR

\begin{tabular}{ll}
\hline Gene & Forward (F) and Reverse (R) primer \\
\hline Wnt10b & F: CCAGGACACGTGGGAATG \\
& R: ATCCAAGAAATCCCGAGAGAAC \\
Wnt5a & F: AGAAACTCTGCCACTTGTATCA \\
& R: GAACTGGTACTGGCACTCTTT \\
$\beta$-catenin & F: CATGTCCTTTGCCACTTTT \\
$\beta$-actin & R: CGCAGCAAAAGAAATCAAC \\
& F: GTGTGGATTGGTGGCTCTAT \\
& R: GACTCTCGTACTCCTGCTTG \\
\hline
\end{tabular}

\section{Evaluation of Alkaline Phosphatase (ALP) in Rat Blood Serum}

The blood samples were taken directly from canthus orbitalis dexter after culling. The blood was added to an anticoagulant and was stirred at a rate of $3000 \mathrm{rpm}$ for 10 min to extract the blood serum. The extracted blood serum was stored and $-80^{\circ} \mathrm{C}$ until analysis. Biochemical analysis by ELISA was performed on all rats $(n=48,24$ rats each for PEMF and control group). ALP as a bone formation marker was measured with the commercially ELISA kit (My Biosources, USA, catalog no MBS011598). The change in absorbance rate was directly proportional to the ALP activity.

\section{Evaluation of Wnt5a, Wnt $10 b$ and $\beta$-catenin in The Bone Callus}

Molecular analysis by RT-qPCR method was performed on 24 of the 48 samples ( $n=12$ each for PEMF and control group). The fractured femur was resected, the callus was then separated from other soft tissues and stored in liquid nitrogen at $-80^{\circ} \mathrm{C}$. Frozen callus was weighed and crushed into powder using a mortar. TRIzol (Life Technologies) was added to the callus powder and mixed until homogeneous in order to obtain maximum RNA. RNA isolation was carried out according to the protocol in the RNeasy kit (Qiagen Cat no. 74104). The primers for Wnt5a, Wnt10b, $\beta$-catenin and Act-Actin are listed in Table 2. Quantification and amplification of target genes by RT-qPCR was performed using QuantiTect SYBR Green PCR (Qiagen). The mRNA expressions were calculated using the Livak method.

\section{Statistical Analysis}

For the analysis of the results, we used the statistical software program SPSS for Windows version 21 (SPSS
Inc., Chicago, Illinois). Data with normal distribution were presented as mean \pm Standard Deviation (SD). The statistically significant effects were detected by one-way Analysis Of Variance (ANOVA) with Tukey's post hoc test. The student's t-test was used for the detection of a difference in Wnt expression level between control and PEMF group. A significant difference is marked as $*(\mathrm{p}<0.05)$.

\section{Results}

\section{HE Staining}

HE-stained histological images of the callus bone in the control and PEMF groups in this study are shown in Fig. 1 to 4 (A\&B with $40 \mathrm{x}$ magnification, $C \& D$ image with $100 \times$ magnification of yellow square area, the bar indicates $1000 \mu$ ).

Histological examination at day 5 in both the control and PEMF groups shows that the fracture gap is still clearly visible and dominated by hematoma, inflammatory cells, fibrin clot, granulation tissue and fibrous tissues with amorphous properties (Fig. 1).

At day 10 of observation, the callus in the PEMF exposure group was dominated by hyaline cartilage in the hypertrophy phase and bone, whereas only fibrous tissue and cartilage was observed in the control group (Fig. 2).

At day 18, the fracture gap was no longer observed; woven bones dominated the external and internal callus. Osteoblasts were aligned on woven bones. In both groups, little cartilage in the hypertrophic phase was observed. This suggests that on the 18th day, the endochondral ossification process is still ongoing (Fig. 3).

At day 28 post-fracture, both control and PEMF groups showed that the fracture gap was dominated by woven bone. This bone will then undergo a remodeling process into lamellar bone, which is characterized by a structured orientation of collagen and the presence of a Haversian system (Fig. 4).

\section{Alkaline Phosphatase Activity in Blood Serum}

The change of ALP activity in blood serum at days 5, 10, 18 and 28 post-fracture is shown in Fig. 5.

In the PEMF exposure group, ALP activity in serum shows a significant increase from days 5 to 28 and from days 10 to 18 . These results indicate that PEMF exposure can accelerate fracture healing, especially in the endochondral phase. 


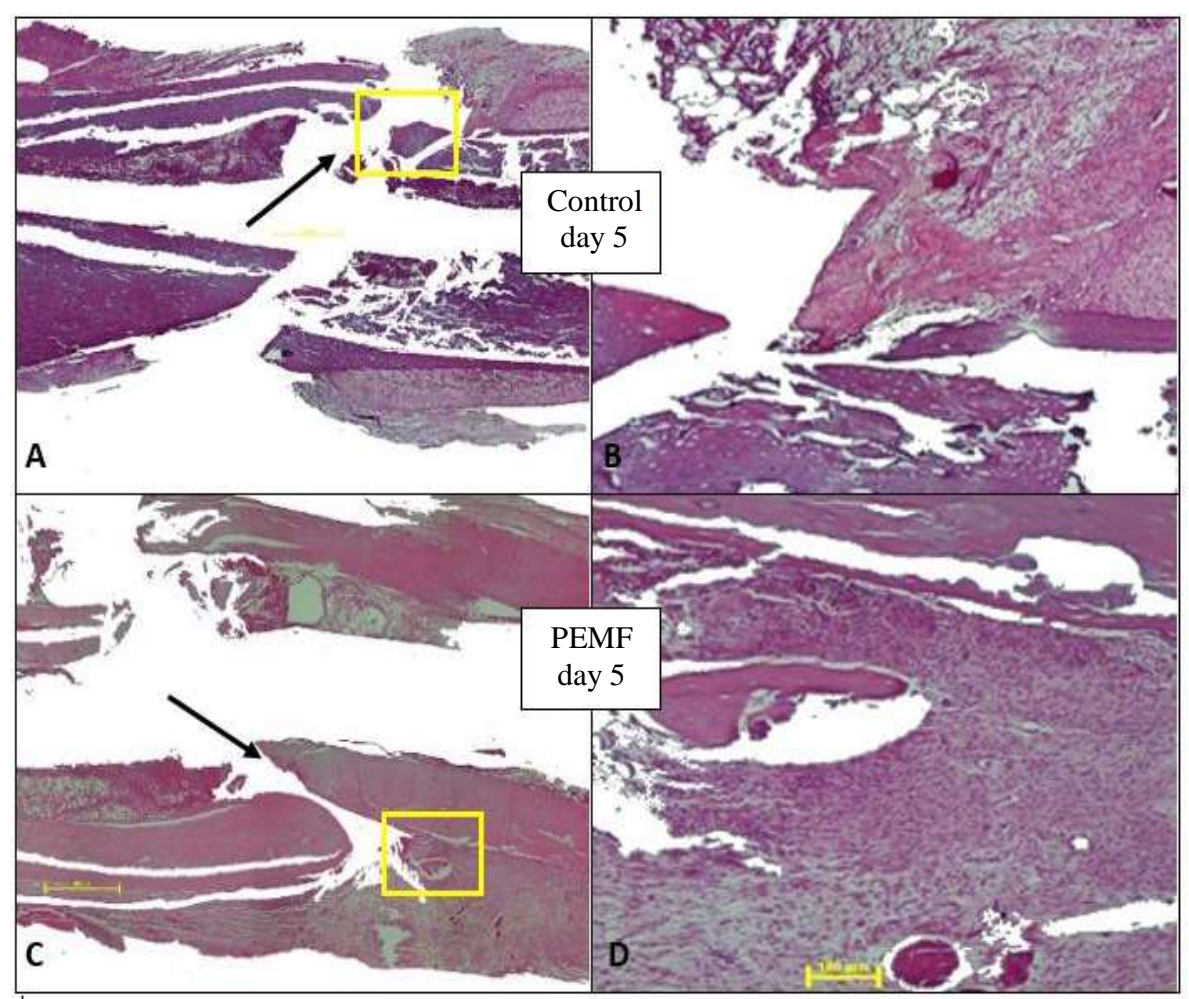

Fig. 1: The histological image of longitudinal sections in both groups at days 5 post-fracture. A black arrow indicates fracture sites. The bar indicates $1000 \mu$. A and B (control group), C and D (PEMF group)

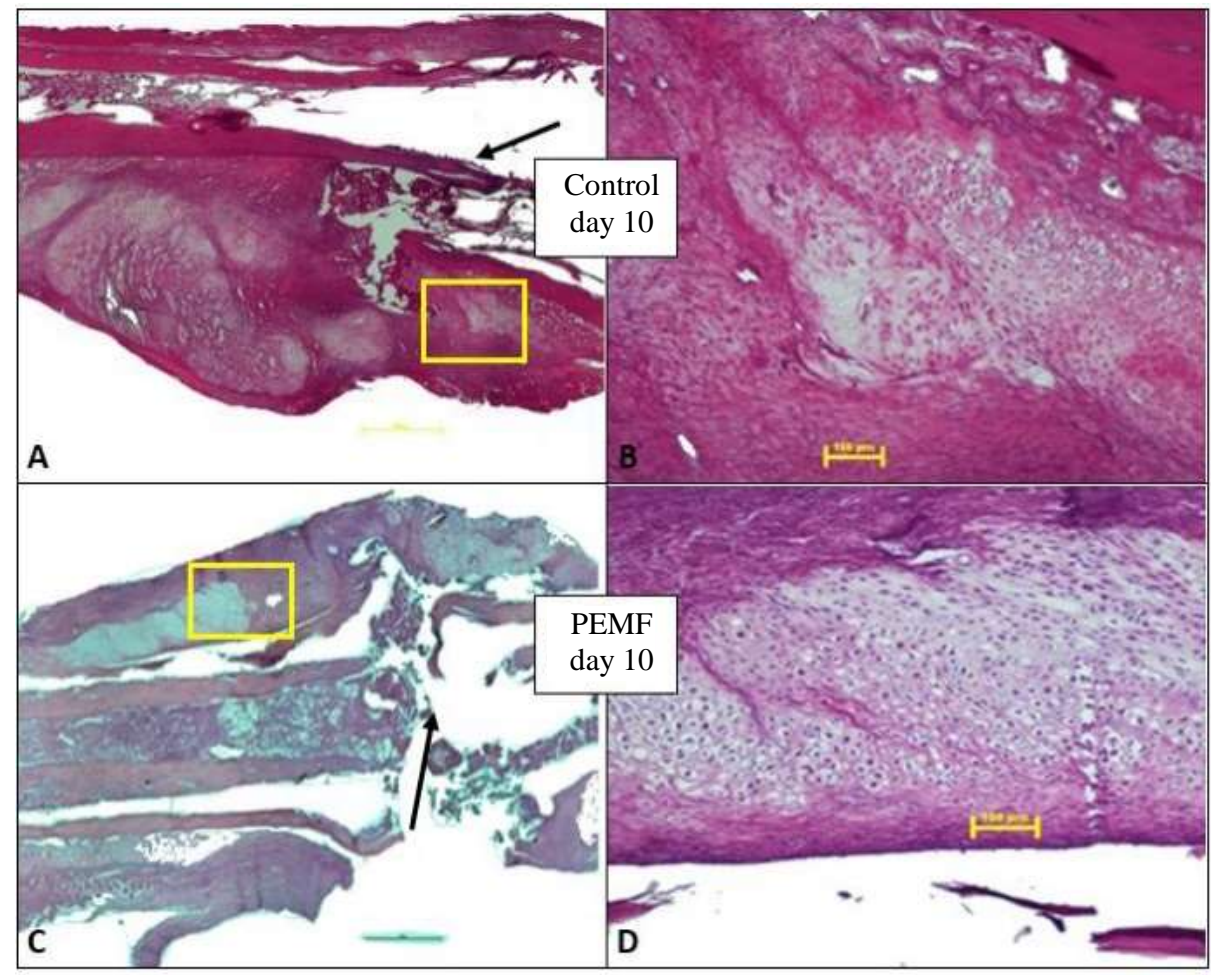

Fig. 2: The histological image of the longitudinal sections with delayed union fracture model in both groups at days 10 post-fracture. Fracture site indicated by a black arrow 
Umiatin U. et al. / OnLine Journal of Biological Sciences 2020, 20 (4): 239.249 DOI: 10.3844/ojbsci.2020.239.249

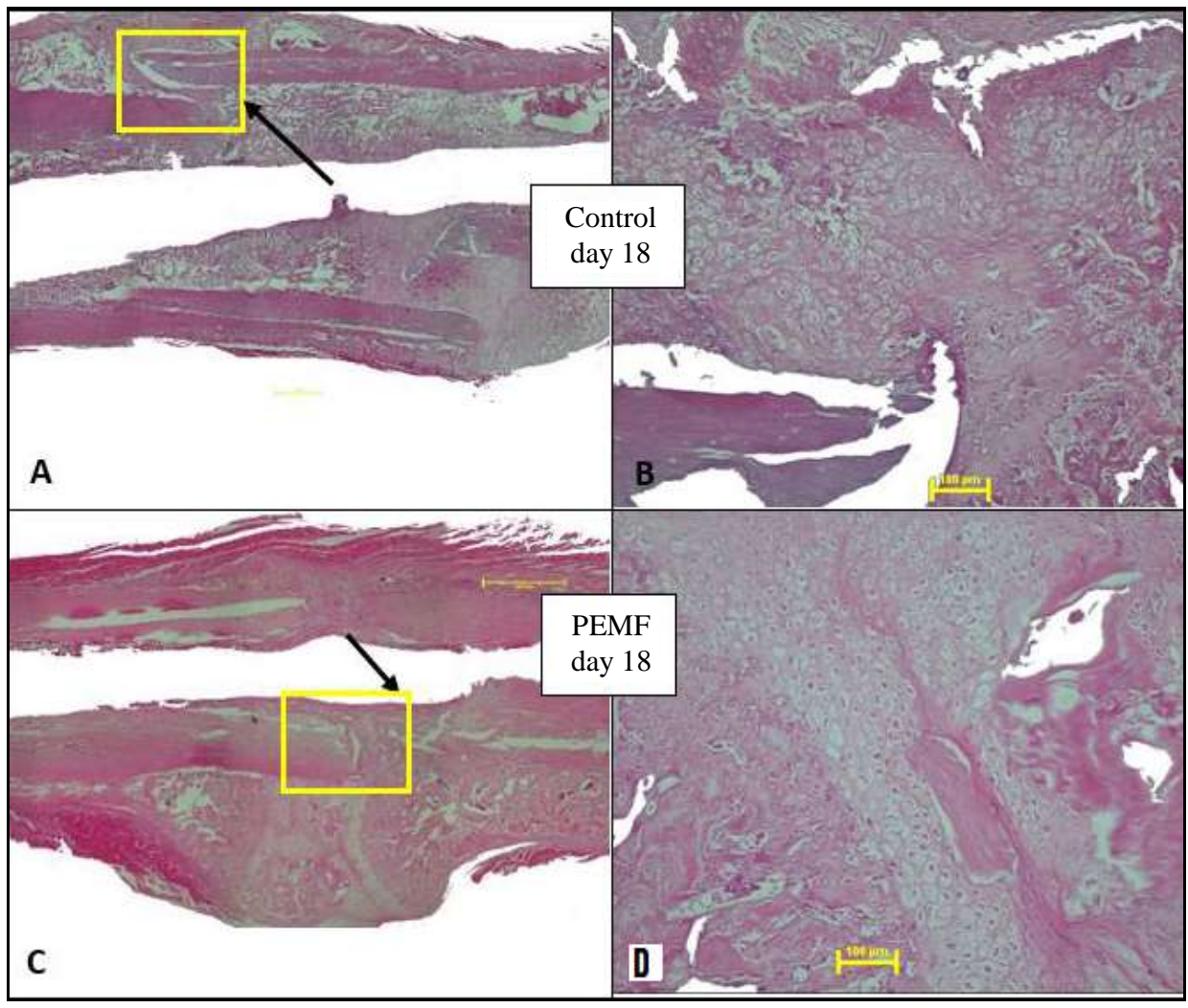

Fig. 3: The histological of longitudinal sections in both groups at days 18 post-fracture. Fracture sites indicated by black arrow

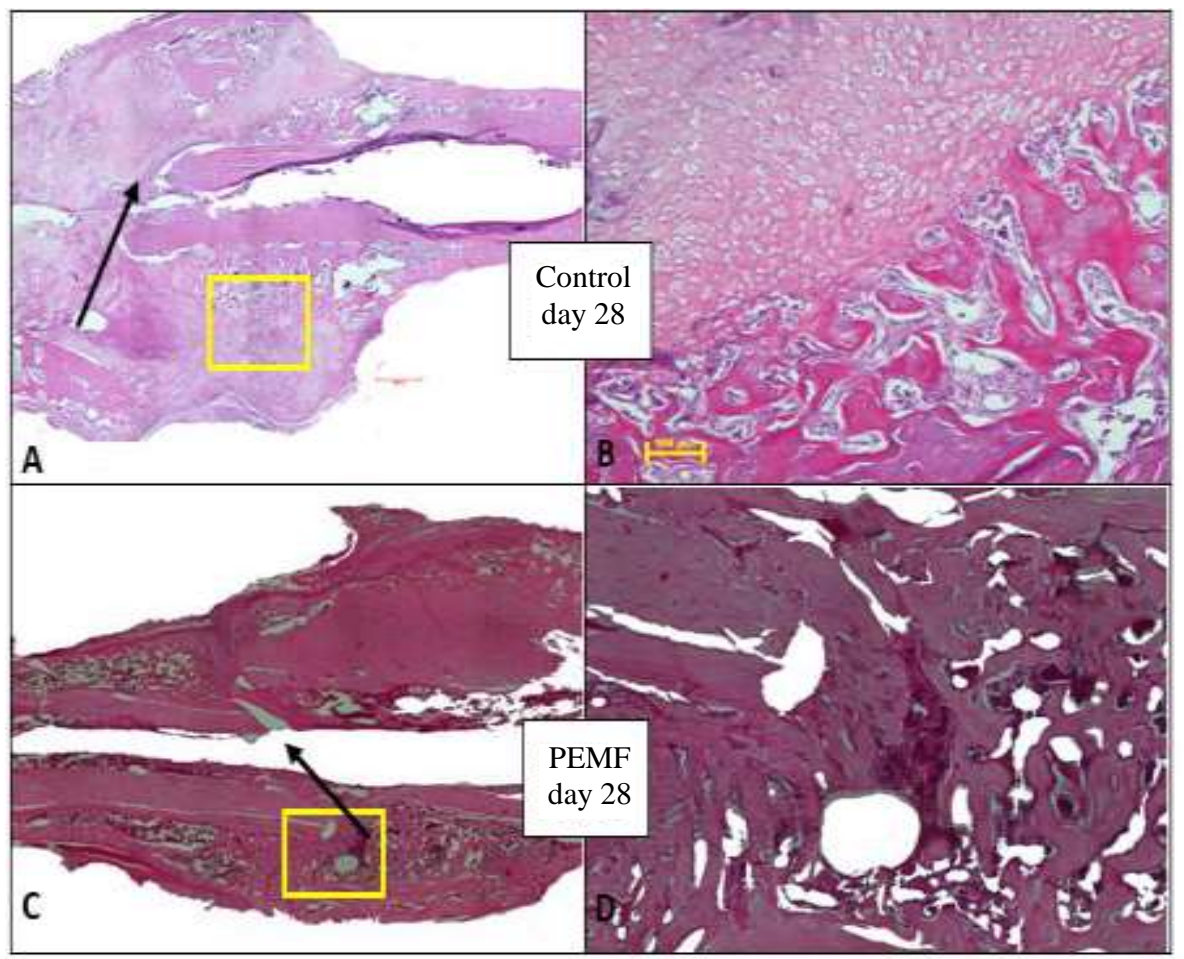

Fig. 4: The histological of longitudinal sections in both groups at days 28 post-fracture. Fracture sites indicated by the black arrow. Bar indicates $1000 \mu$ 


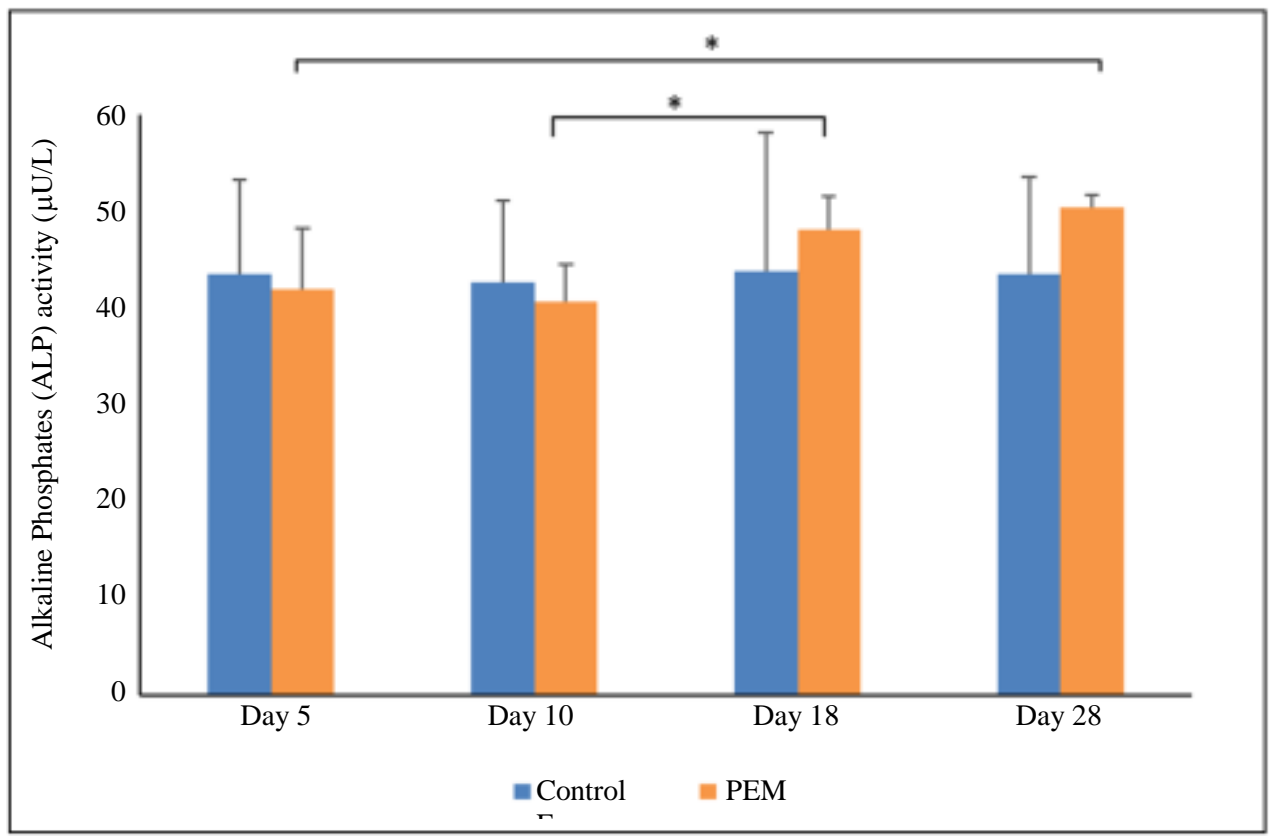

Fig. 5: ALP activity in both control and PEMF group at days 5, 10, 18 and 28. PEMF can significantly increase the activity of ALP between day 10 and day 18 post-fracture. Data were presented as a mean \pm standard deviation

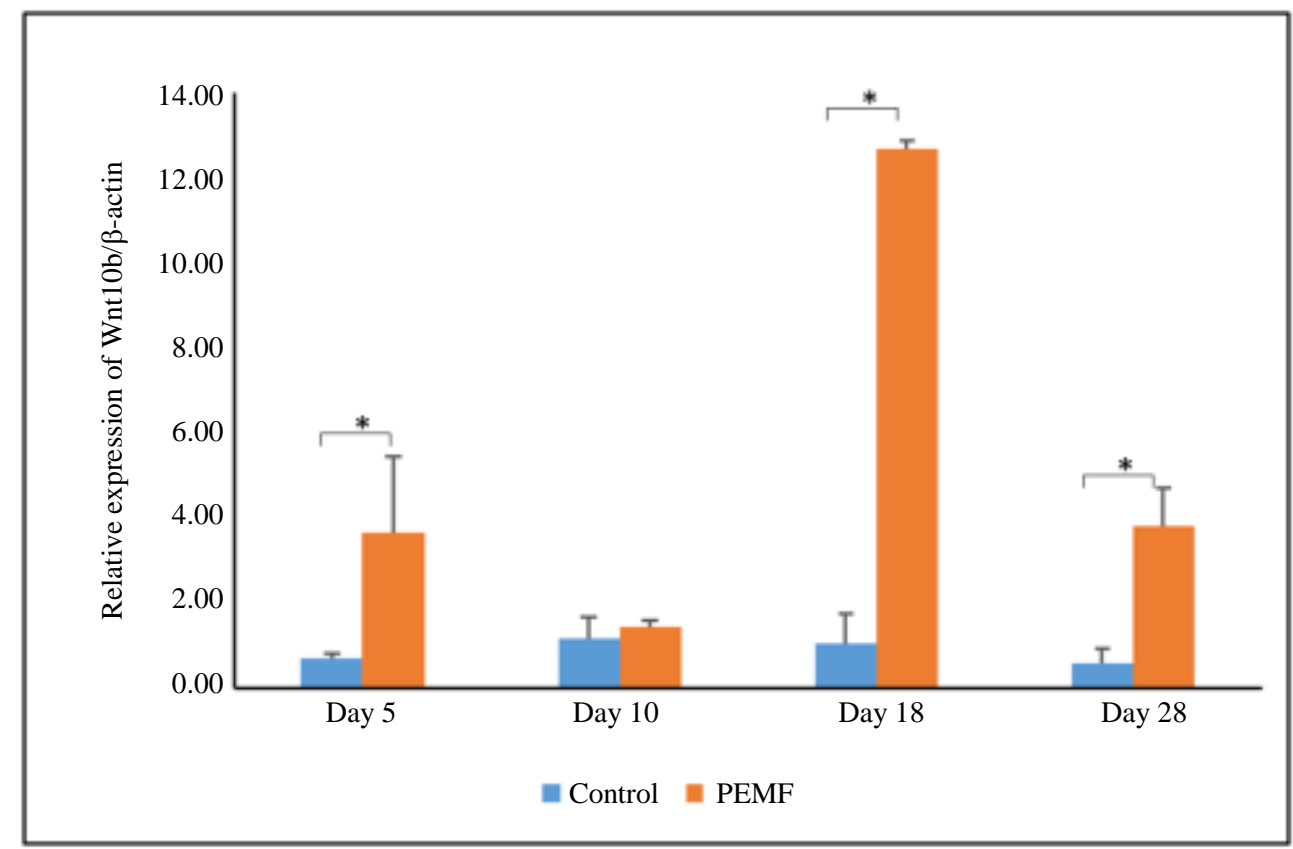

Fig. 6: Wnt10b expression level relative to $\beta$-actin (ACTB) of the healing process over time. *indicates a statistically significant difference between the control and PEMF group $(\mathrm{p}<0.05)$

\section{Expression of Wnt10b, Wnt $5 a$ and $\beta$-Catenin}

Fracture healing involves several signaling pathways, including Wnt signaling pathway. The relative expression of Wnt10b, Wnt5a and $\beta$-catenin relative to $\beta$-actin are shown in Fig. 6 to 8. The PEMF group has shown the higher relative expression of Wnt10 b, Wnt5a and $\beta$-catenin than in the control group. The highest relative expression of Wnt10b, Wnt5a and $\beta$-catenin occurred on the day 18 post fracture. Furthermore, on the day 28 there were a decrease in the expression of these gene. 


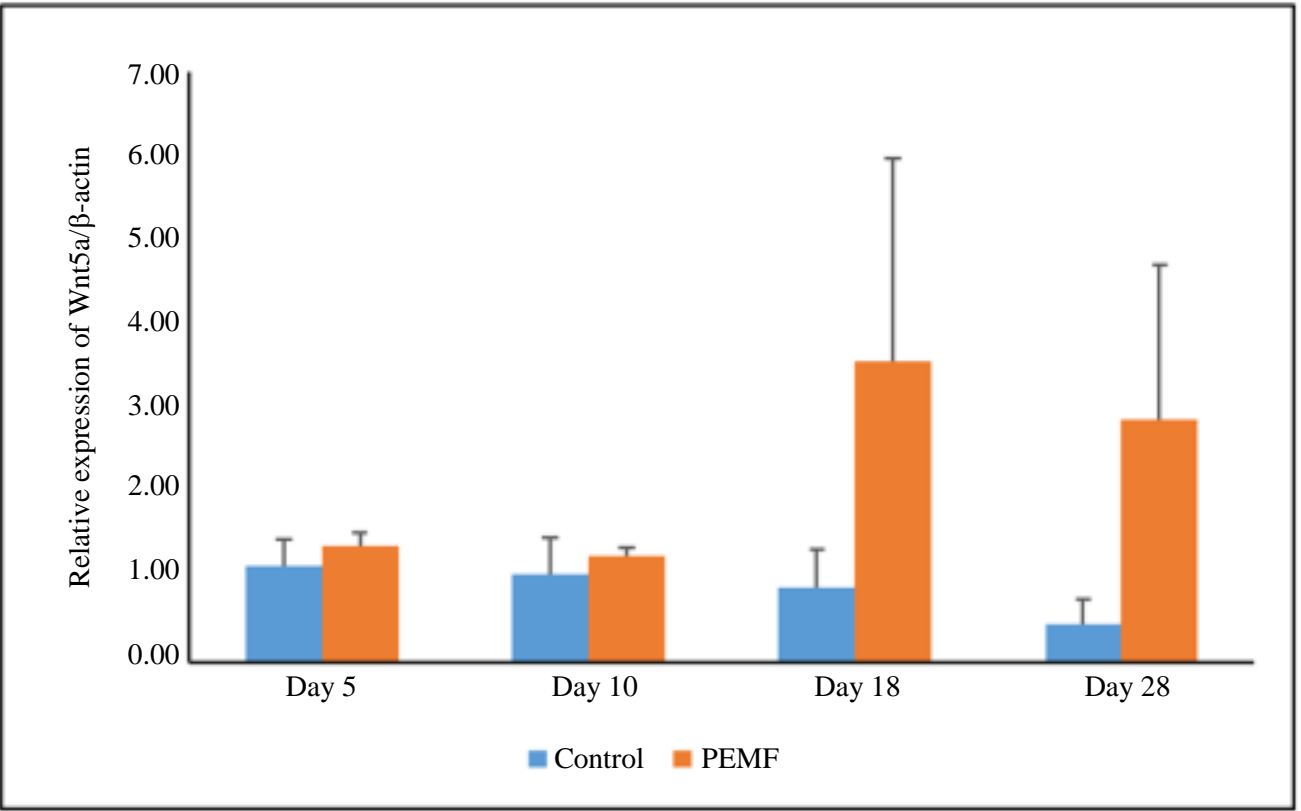

Fig. 7: Wnt5a expression level relative to $\beta$-actin (ACTB) of the healing process over time. *indicates a statistically significant difference between the control and PEMF group $(\mathrm{p}<0.05)$

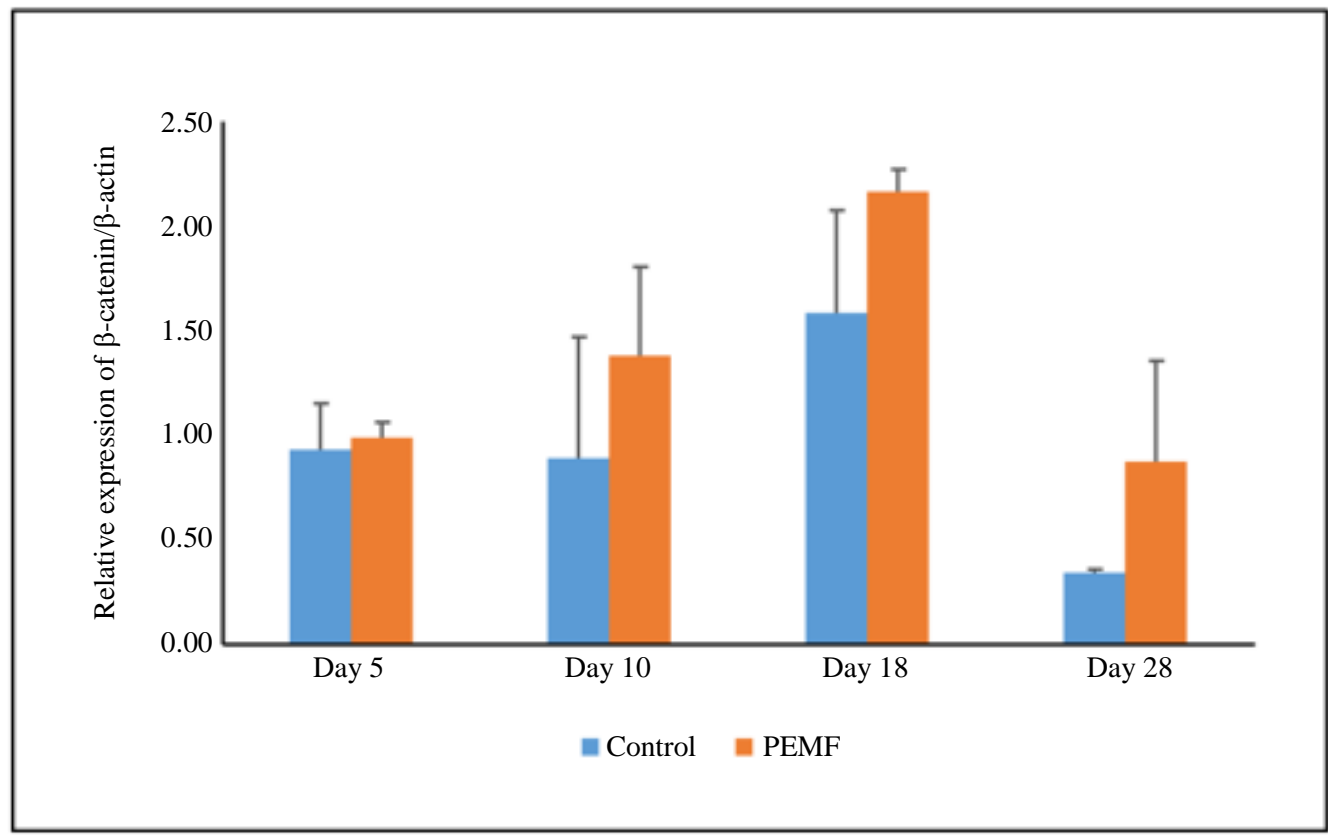

Fig. 8: $\beta$-catenin expression level relative to $\beta$-actin $(\mathrm{ACTB})$ of the healing process over time. *indicates a statistically significant difference between the control and PEMF group $(\mathrm{p}<0.05)$

\section{Discussion}

Fracture healing consists of four overlapping phases, starting with the inflammatory response, soft callus formation, hard callus formation (woven bone) and the remodeling phase. Disruption in the healing phase could cause delayed union (Hak et al., 2014).
Inflammation is a very crucial early phase during normal fracture healing. This phase is marked by the presence of a hematoma that acts as a source for various signaling molecules needed to initiate the healing process. The delayed presence of a hematoma led to delays in cartilage formation (Peters et al., 2010). The final inflammatory phase and the beginning of the 
reparative phase occurred at day 5 of the first-week post-fracture (Vieira et al., 2015). At day 10, hyaline cartilage in the proliferation phase was observed in the fracture gap. This suggests that PEMF exposure accelerates the formation of cartilage at day 10 postfracture. These results are in agreement with a study conducted by (Li et al., 2017) which concluded that in the second-week post-fracture, chondroblasts had reached the hypertrophy phase.

Fracture healing is commonly assessed clinically and radiologically, but it is difficult to distinguish early delayed union from nonunion fractures. Biochemical markers, specifically Alkaline Phosphatase (ALP) has been reported to reflect osteoblastic activity, bone matrix formation and mineralization. It could be a useful tool for predicting whether fractures are at risk of developing a nonunion. ALP is a hydrolase enzyme in the cell membrane, of which the highest concentration is found in osteoblasts. The major source of ALP in plasma and serum is in bone and liver. ALP is thought to also play a role in suppressing osteoblast apoptosis, increasing osteoblast proliferation and mineralization. Jing et al. (2013) reported that ALP in rat serum decreased after PEMF intervention for 12 weeks.

In the PEMF exposure group, we found a significant increase in ALP activity in serum at days 5 to 28 and days 10 to 18 . Serial ALP tests assist physicians in predicting progress as well as the outcome of fracture healing. In this study, the highest activity of ALP occurred on the 28th-day postfracture, amounting $(50.47 \pm 1.28)$ IU/L. Another study reported that ALP activity would reach its peak in week 3 and decrease at week 4 post-fracture (Jing et al., 2013). There is a difficulty in getting consistent ALP values in different studies and this may be due to different rat strains, animal handling techniques and differences in fracture types in rats.

Wnt10b is a gene involved in the canonical signaling pathway. Canonical Wnt signaling is the main regulator of bone mass homeostasis (Jing et al., 2013). Hill et al. (2005) reported that canonical Wnt/ $\beta$-catenin signaling was essential for skeletal lineage differentiation, preventing the trans differentiation of osteoblastic cells into chondrocytes. The proliferation of osteoblasts was reduced when Wnt signaling was blocked and proliferation is increased when Wnt signaling was constitutively active (Kim and Leopold, 2012). Wnt10b showed greater expression in the injured bone marrow than in normal rat (Congdon et al., 2008). The Wnt5a is a gene that plays a role in the noncanonical Wnt signaling pathway. This pathway is independent of $\beta$-catenin. The Wnt5a gene is expressed in bone marrow, both in normal and injured conditions (Congdon et al., 2008). Wnt5a is capable both in inducing and repressing $\beta$-catenin signaling pathway, depending on the time, site of expression and the type of receptor present on the surface of the cell membrane (van Amerongen et al., 2012). $\beta$-catenin is known to be a major regulator in bone development at the embryonic stage. At day 10 , the expression of $\beta$-catenin in the PEMF exposure group was higher than in the control group. In this phase mesenchymal cells begin to differentiate into osteoblasts and chondrocytes. This suggests that $\beta$-catenin expression is needed at the stage of chondrogenesis and osteogenesis differentiation. Expression of $\beta$-catenin during chondrogenesis encourages the differentiation of pre-chondrocytes to hypertrophic chondrocytes. An increase in $\beta$-catenin expression differentiation also plays a role in encouraging the transdifferentiation of hypertrophic chondrocytes to osteoblasts. $\beta$-catenin expression at this stage prevents differentiation of mesenchymal cells toward chondrocytes by suppressing Sox 9 expression. Overexpression of Sox 9 could cause an abundance of hypertrophic chondrocytes resulting in delayed union (Hu et al., 2017).

In recent studies, the highest Wnt10b expression occurred at day 18 post-fracture. These results indicate that exposure to PEMF encourages osteoblast activity in the mineralizing cartilage matrix, reflected by the highest expression of Wnt10b on day 18. The increase in Wnt $10 \mathrm{~b}$ gene expression may indicate that PEMF stimulation promotes the commitment of stem cells towards osteoblastic differentiation. Wnt10b is also expressed by mature osteoclasts, encouraging migration of mesenchymal cells to the remodeling area and encouraging differentiation of mesenchymal cells into osteoblasts (Pederson et al., 2008). On day 28, there was a decrease in Wnt10b, Wnt5a and $\beta$-catenin expression in both the PEMF control groups. The results of this study are in accordance with research conducted by (Chen et al., 2007) which reported a decrease in $\beta$ catenin expression at week 4 after fracture.

Our findings showed that on day $18, \beta$-catenin expression was higher compared to days 5, 10 and 28, both in the control and exposed groups. $\beta$-catenin activity decreased in the final stage of osteoblasts. It was differentiating into osteocytes at the time of mineralization of the bone matrix. Expression of $\beta$-catenin also played a role in regulating osteoclastogenesis by suppressing the expression of pro-osteoclast factor Rank (Houben et al., 2016). In the remodeling phase, a decrease in $\beta$-catenin serves to prevent osteoclast precursors from proliferating and encourages the differentiation of pre-osteoclasts into mature osteoclasts that absorb bone. Overexpression of $\beta$-catenin in the remodeling phase can cause interference with osteoclast differentiation (Dao et al., 2012). Osteoblast regulates osteoclast differentiation and function by secreting Rankl and OPG. Both are regulated by the canonical Wnt which promotes bone formation and suppress bone resorption. The previous study by (Sari et al., 2019) has reported that PEMF exposure increase the expression of Rank, Rankl 
and OPG in the rat with delayed union fracture model. The high expression of these genes was due to the differentiation of osteoblast.

The results of this study are in accordance with a study conducted by (Chen et al., 2007) which showed a decrease in $\beta$-catenin expression at week 4 postfracture. Expression of $\beta$-catenin during condensation encourages differentiation of pre-chondrocytes into hypertrophic chondrocytes. Also, the increase in $\beta$ catenin expression had a role in encouraging the transdifferentiation of hypertrophic chondrocytes into osteoblasts. The expression of $\beta$-catenin at this stage prevents the differentiation of mesenchymal cells towards chondrocytes by suppressing Sox9 expression (Hu et al., 2017).

\section{Conclusion}

The present in vivo study reported that PEMF stimulation accelerated bone fracture healing in the early stages associated with the time of resorption of fibrous tissue and increasing the activity of osteoblasts, which is characterized by an increase in alkaline levels in blood serum. Based on the results of our study, we conclude that in a rat femur delayed union fracture model, the expression of Wnt5a, Wnt10b and $\beta$-catenin was higher in rats exposed to PEMF stimulation. Overall, the expression of Wnt-10b was higher than Wnt-5a, suggesting that canonical Wnt signaling could positively regulate bone formation. This finding may have implications in the treatment of delayed fracture healing using PEMF stimulation by promoting Wnt10b, Wnt5a and $\beta$-catenin in the bone callus of rats. PEMF may be valuable for the intervention of delayed union fracture in the clinical setting.

\section{Funding Information}

This study was supported by the doctoral research grant from Universitas Indonesia, No. 1309/UN2.R3.1/HKP.05.00/2018

\section{Author's Contributions}

Umiatin, U.: Designing and conducting experiments, aacquisition of data, analyzing data, drafting the article.

Ismail Hadisoebroto Dilogo, Puji Sari and Sastra Kusuma Wijaya: Designing experiments, reviewing the article critically for significant intellectual content, give final approval of the submitted and revised article.

\section{Ethics}

This article has not been published or presented elsewhere in part or in entirely and is not under consideration by another journal. All the authors have approved the manuscript Authors should address any ethical issues that may arise after the publication of this article.

\section{References}

Bao, Q., Chen, S., Qin, H., Feng, J., Liu, H., Liu, D., ... \& Zong, Z. (2017). An appropriate Wnt/ $\beta$-catenin expression level during the remodeling phase is required for improved bone fracture healing in mice. Scientific Reports, 7(1), 1-11.

Ceccarelli, G., Bloise, N., Mantelli, M., Gastaldi, G., Fassina, L., Cusella De Angelis, M. G., ... \& Visai, L. (2013). A comparative analysis of the in vitro effects of pulsed electromagnetic field treatment on osteogenic differentiation of two different mesenchymal cell lineages. BioResearch open access, 2(4), 283-294.

Chen, Y., \& Alman, B. A. (2009). Wnt pathway, an essential role in bone regeneration. Journal of cellular biochemistry, 106(3), 353-362.

Chen, Y., Whetstone, H. C., Lin, A. C., Nadesan, P., Wei, Q., Poon, R., \& Alman, B. A. (2007). Betacatenin signaling plays a disparate role in different phases of fracture repair: implications for therapy to improve bone healing. PLoS Med, 4(7), e249.

Choi, M. C., Cheung, K. K., Li, X., \& Cheing, G. L. Y. (2016). Pulsed electromagnetic field (PEMF) promotes collagen fibre deposition associated with increased myofibroblast population in the early healing phase of diabetic wound. Archives of dermatological research, 308(1), 21-29.

Congdon, K. L., Voermans, C., Ferguson, E. C., DiMascio, L. N., Uqoezwa, M., Zhao, C., \& Reya, T. (2008). Activation of Wnt signaling in hematopoietic regeneration. Stem cells, 26(5), 1202-1210.

Dao, D. Y., Jonason, J. H., Zhang, Y., Hsu, W., Chen, D., Hilton, M. J., \& O'Keefe, R. J. (2012). Cartilage-specific $\beta$-catenin signaling regulates chondrocyte maturation, generation of ossification centers and perichondrial bone formation during skeletal development. Journal of Bone and Mineral Research, 27(8), 1680-1694.

Dilogo, I. H., Djaja, A. D., \& Tendean, R. H. (2018). The effect of extreme low frequency-pulse electromagnetic field exposure in the healing process of sprague dawley mouse delayed union femur fracture: Study of rust radiology score. Asian Journal of Microbiology, Biotechnology and Environmental Sciences, 20(December), S74-S78. 
Fauzi, A., Kamal, A. F., Kurniawan, A., \& Kodrat, E. (2015). Role of sildenafil in acceleration of delayed union fracture healing on Sprague-Dawley rats model. Br J Med Med Res, 8, 419-428.

Fu, Y. C., Lin, C. C., Chang, J. K., Chen, C. H., Tai, I. C., Wang, G. J., \& Ho, M. L. (2014). A novel single pulsed electromagnetic field stimulates osteogenesis of bone marrow mesenchymal stem cells and bone repair. PloS one, 9(3), e91581.

Gimble, J.M., Zvonic, S., Flyod, Z.E., Kaseem, M., Nuttall, M.E. (2006). Playing with bone and fat. J Cell Biochem 98 :251-266.

Hak, D. J., Fitzpatrick, D., Bishop, J. A., Marsh, J. L., Tilp, S., Schnettler, R., ... \& Alt, V. (2014). Delayed union and nonunions: epidemiology, clinical issues and financial aspects. Injury, 45, S3-S7.

Heilmann, A., Schinke, T., Bindl, R., Wehner, T., Rapp, A., Haffner-Luntzer, M., ... \& Ignatius, A. (2013). The Wnt serpentine receptor Frizzled-9 regulates new bone formation in fracture healing. PloS one, 8(12), e84232.

Hill, T. P., Später, D., Taketo, M. M., Birchmeier, W., \& Hartmann, C. (2005). Canonical Wnt/ $\beta$-catenin signaling prevents osteoblasts from differentiating into chondrocytes. Developmental cell, 8(5), 727-738.

Houben, A., Kostanova-Poliakova, D., Weissenböck, M., Graf, J., Teufel, S., von der Mark, K., \& Hartmann, C. (2016). $\beta$-catenin activity in late hypertrophic chondrocytes locally orchestrates osteoblastogenesis and osteoclastogenesis. Development, 143(20), 3826-3838.

Hu, D. P., Ferro, F., Yang, F., Taylor, A. J., Chang, W., Miclau, T., ... \& Bahney, C. S. (2017). Cartilage to bone transformation during fracture healing is coordinated by the invading vasculature and induction of the core pluripotency genes. Development, 144(2), 221-234.

Jing, D., Li, F., Jiang, M., Cai, J., Wu, Y., Xie, K., ... \& Shen, G. (2013). Pulsed electromagnetic fields improve bone microstructure and strength in ovariectomized rats through a $\mathrm{Wnt} / \mathrm{Lrp} 5 / \beta$-catenin signaling-associated mechanism. PLoS One, 8(11), e79377.

Kim, P. H., \& Leopold, S. S. (2012). Gustilo-Anderson Classification.

Kokubu, T., Hak, D. J., Hazelwood, S. J., \& Reddi, A. H. (2003). Development of an atrophic nonunion model and comparison to a closed healing fracture in rat femur. Journal of Orthopaedic Research, 21(3), 503-510.
Kooistra, B. W., Jain, A., \& Hanson, B. P. (2009). Electrical stimulation: nonunions. Indian Journal of Orthopaedics, 43(2), 149.

Li, X., Ren, W., Wang, F., Zhu, Y., Wang, H., \& Liu, J. (2017). The study of factors that affects the fracture healing in diabetic rats.

Liu, H., Abbott, J., \& Bee, J. A. (1996). Pulsed electromagnetic fields influence hyaline cartilage extracellular matrix composition without affecting molecular structure. Osteoarthritis and Cartilage, 4(1), 63-76.

Marcellini, S., Henriquez, J. P., \& Bertin, A. (2012). Control of osteogenesis by the canonical Wnt and BMP pathways in vivo: cooperation and antagonism between the canonical Wnt and BMP pathways as cells differentiate from osteochondroprogenitors to osteoblasts and osteocytes. Bioessays, 34(11), 953-962.

Pederson, L., Ruan, M., Westendorf, J. J., Khosla, S., \& Oursler, M. J. (2008). Regulation of bone formation by osteoclasts involves Wnt/BMP signaling and the chemokine sphingosine-1phosphate. Proceedings of the National Academy of Sciences, 105(52), 20764-20769.

Peters, A., Schell, H., Bail, H. J., Hannemann, M., Schumann, T., Duda, G. N., \& Lienau, J. (2010). Standard bone healing stages occur during delayed bone healing, albeit with a different temporal onset and spatial distribution of callus tissues. Histology and histopathology, Vol. 25, no 9.

Sari, P., Reihannisha, I., Umiatin, U., Suryandari, D.A., Yunaini, L. (2019). Expression relative of Rank, Rankl and OPG gene on at femoral fracture healing process in delayed union model after pulsed electromagnetic field exposure. Journal of Global Pharma Technology, Vol.11, Issue 04 (Suppl.), 223-230.

Sari, P., Umiatin, Yunaini, L., Luviah, E., Mazfufah, N.F. (2020). Relative expression of PPAR $\gamma$ and Adipoq mRna as marker of inhibition Adipogenesis on Mesenchymal Stem Cells after Pulsed Electromagnetic Field (PEMF) Exposure. Journal of Global Pharma Technology, Vol. 12, Issue 01 (Suppl.), 307-317.

Shi, H. F., Xiong, J., Chen, Y. X., Wang, J. F., Qiu, X. S., Wang, Y. H., \& Qiu, Y. (2013). Early application of pulsed electromagnetic field in the treatment of postoperative delayed union of longbone fractures: a prospective randomized controlled study. BMC Musculoskeletal Disorders, 14(1), 35. 
Umiatin, Dilogo, I. H., Wijaya, S. K., Sari, P., \& Djaja, A. D. (2019, April). Design and development of pulse electromagnetic fields (PEMF) as adjuvant therapy for fracture healing: A preliminary study on rats. In AIP Conference Proceedings (Vol. 2092, No. 1, p. 020028). AIP Publishing LLC.

Van Amerongen, R., Fuerer, C., Mizutani, M., \& Nusse, R. (2012). Wnt5a can both activate and repress Wnt/ $\beta$-catenin signaling during mouse embryonic development. Developmental biology, 369(1), 101-114.
Vieira, A. E., Repeke, C. E., Junior, S. D. B. F., Colavite, P. M., Biguetti, C. C., Oliveira, R. C., ... \& Garlet, G. P. (2015). Intramembranous bone healing process subsequent to tooth extraction in mice: microcomputed tomography, histomorphometric and molecular characterization. PloS one, 10(5), e0128021.

Zhang, X., Liu, X., Pan, L., \& Lee, I. (2010). Magnetic fields at extremely low-frequency $(50 \mathrm{~Hz}, 0.8 \mathrm{mT})$ can induce the uptake of intracellular calcium levels in osteoblasts. Biochemical and biophysical research communications, 396(3), 662-666. 